\title{
Lessons Learned from An Implant-Related Infection with Bacillus Spp of the Proximal Femur: A Rare and Insidious Complication After Internal Fixation of Closed Fractures
}

\author{
Sebastian Ebert ${ }^{1}$, Christoph Meier ${ }^{1}$, Severin Meili $^{2}$ and Peter Wahl ${ }^{1 *}$ \\ ${ }^{1}$ Division of Orthopaedics and Traumatology, Cantonal Hospital Winterthur, Winterthur, Switzerland \\ ${ }^{2}$ Division of Traumatology, Traumatology Hospital Uster, Uster, Switzerland
}

*Corresponding author: Peter Wahl, Division of Orthopaedics and Traumatology, Cantonal Hospital Winterthur, Brauerstrasse 15, 8401 Winterthur, Switzerland

\section{ARTICLE INFO}

Received: 幽 June 19, 2019

Published: July 01, 2019

Citation: Sebastian Ebert, Christoph Meier, Severin Meili, Peter Wahl. Lessons Learned from An ImplantRelated Infection with Bacillus Spp of the Proximal Femur: A Rare and Insidious Complication After Internal Fixation of Closed Fractures. Biomed J Sci \& Tech Res 19(2)-2019. BJSTR. MS.ID.003274.

\section{ABSTRACT}

Background: Bacillus are Gram-positive, primarily aerobic, rod-shaped and sporeforming bacteria. Ubiquitary in environment, infections with Bacillus are known to occur after open fractures but are rarely described after internal fixation of closed fractures. Based on a case of implant-related infection of the proximal femur with Bacillus spp after internal fixation, we report on the associated diagnostic and therapeutic challenges.

Methods: Case report with review of the essential features.

Results: A 74-year-old male suffered a pertrochanteric fracture of the femur due to a low energy fall. Internal fixation was performed with a cephalomedullary nail. Three debridements were performed for postoperative wound oozing before infection with Bacillus spp was detected. Insufficient sampling with misinterpretation and empiric antibiotic treatment delayed proper diagnosis. One-stage implant exchange, local application of antibiotic-loaded calcium sulphate, combined with systemic antibiotic treatment, healed the infection and the fracture. Due to possible spore formation, the antibiotic treatment was maintained for a total of six months. No recurrence of the infection was observed, now over two years later.

Conclusion: Orthopaedic or trauma implant-related infections with Bacillus spp are reported rarely, except after open fractures. It usually presents with large seroma, recurrent hematomas or wound oozing. Whenever revising an implant, proper sampling with four to six biopsies is recommended, to avoid misinterpretation and subsequent wrong management, as low virulence microorganisms may cause local infection with few or subtle clinical signs only. Extended duration of antibiotic treatment should be considered for sporulating bacteria, with the aim of reducing the risk of late recurrence.

\section{Case Report}

A 74 years old male, known for chronic alcohol abuse, suffered a trochanteric fracture of his left femur following a low energy fall (Figure 1A). Internal fixation with a long cephalomedullary nail (Proximal Femoral Nail Antirotation, DePuy Synthes, Zuchwil, Switzerland) and subtrochanteric cerclage wires was performed within 24 hours (Figure 1B). The patient was discharged home on the 6th day after surgery, fully weight-bearing with crutches. The further initial postoperative course was uneventful. However, five weeks postoperatively the patient had to be readmitted, due to local swelling and wound oozing, associated with elevated white blood count (WBC, $11.4 \mathrm{G} / \mathrm{l}$ ) and CRP (68 mg/l). The body core temperature was normal. Due to a tonsillitis, an antibiotic treatment with amoxicillin/clavulanate orally had been started four days before by his general practitioner. A wound revision was performed on the day of admission including debridement, irrigation and primary wound closure. Two deep tissue biopsies 
were sampled for microbiological workup (aerobic and anaerobic cultures, including enrichment broth, with 14 days incubation). All the hardware was retained. Postoperatively, amoxicillin/clavulanate was administered parenterally. Due to persistent wound secretion and persistent elevation of the inflammatory parameters, wound revision was repeated five and nine days later.
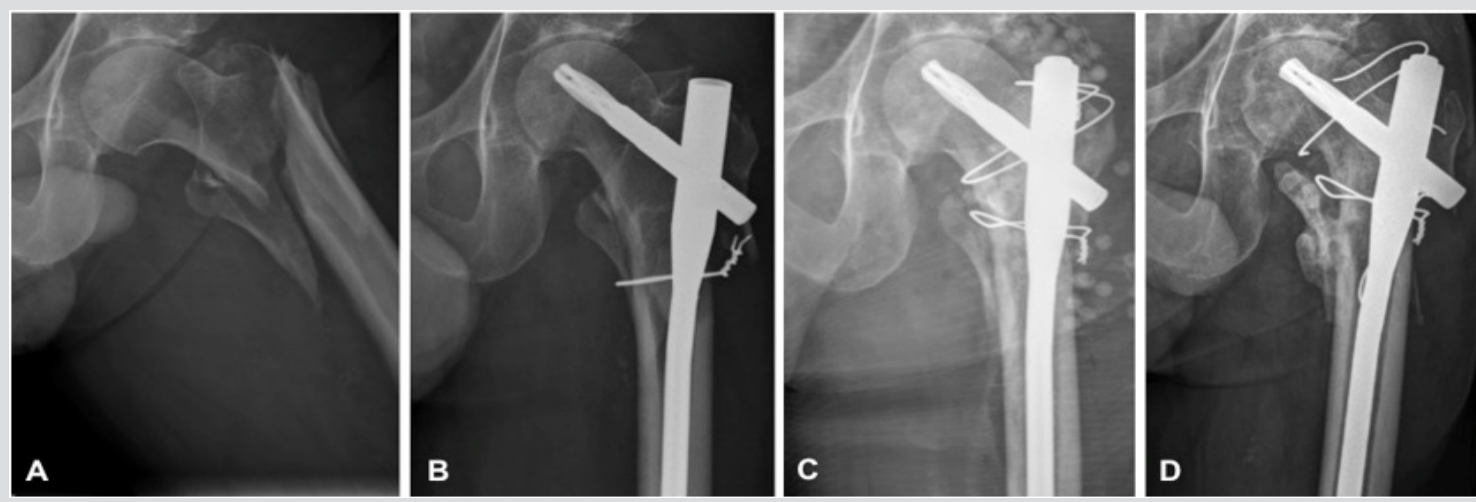

Figure 1: Picture A shows the initial trochanteric fracture of the left femur after a low-energy fall. Internal fixation with a subtrochanteric cerclage and a long cephalomedullary nail was performed urgently (Figure B). Due to an implant-related infection with Bacillus spp, debridement, nail-exchange, application of local antibiotics with calcium sulphate pellets loaded with vancomycin, and refixation of the greater trochanter with wire cerclages was performed (Figure C). After 5 months, breakage of the cranial cerclage and secondary dislocation of the greater trochanter was seen (Figure D). The patient however complained only of minimal left sided hip pain.

Three, respectively two microbiological tissue biopsies were sampled on these occasions. Bacillus spp was detected for the first time in two of two samples from the third debridement, whereas all the previous samples failed to detect any microorganisms at all. However, these positive samples had been discarded as contamination and the antibiotic treatment was discontinued. The patient was discharged nine days after the last operation, despite persisting minor secretion from the former suction drain port The wound from the surgical approach presented dry without any signs of wound healing disturbances. At discharge, leucocytes had normalized, but the CRP remained elevated at $77 \mathrm{mg} / \mathrm{l}$. However, three days after discharge, the patient had to be readmitted with a breakdown of the wound with serous discharge. The WBC was elevated again, at $10.2 \mathrm{G} / \mathrm{l}$, and the CRP had increased to 101 $\mathrm{mg} / \mathrm{l}$, but there was no elevated temperature. Another revision was performed the same day, with debridement, removal of the cerclage, and drainage. Intraoperatively, three biopsies had been sampled for microbiological workup, but this time before any interfering antibiotic treatment. Intravenous antibiotic treatment with amoxicillin/clavulanate was re-established postoperatively.

One tissue sample showed growth of Bacillus spp. Now the infection was diagnosed, integrating the results from the previous revisions, with Bacillus spp being identified in three separate samples, fulfilling even the more rigorous historical diagnostic criteria for low-virulence implant-associated infections [1]. MALDITOF identification could not differentiate between B. cereus and B. thurigensis. Antibiotic resistance was identified against penicillin only, but no other antibiotic drug. In the presence of an implant-associated infection more than six weeks after the initial fracture stabilization, retention of the implants was considered inappropriate. Thus, a single-stage nail exchange with a meticulous debridement and reaming of the femoral medullary cavity was performed. The greater trochanter was refixated with several cerclage wires. Furthermore, two resorbable bead kits of calcium sulphate pellets (Osteoset standard cure $25 \mathrm{cc}$, Wright Medical, Arlington, TN, USA) loaded with $4 \mathrm{~g}$ vancomycin in total, were placed in the wound cavity around the implant as local antibiotic application (Figure 1C) [2-4].

This time, six biopsies for microbiological workup, and additional biopsies for histology, were sampled. Despite ongoing antibiotic treatment with amoxicillin/clavulanate, Bacillus spp was identified again in one sample. Antibiotic treatment was continued orally with levofloxacin, following recommendations from consulting infectious diseases specialists. Postoperatively, the inflammatory parameters normalized within a few days. Mobilization with partial weight bearing was uneventful. The patient was discharged one week after the last operation, despite persistent slight serous secretion of the wound. One week later, the wound presented inconspicuous and dry, allowing then the administration of rifampin in addition to levofloxacin. This addition was made with the intention to enhance treatment efficacy, as a particularly low minimal inhibition concentration (MIC) was identified for this antibiotic agent. Six weeks after the last revision, signs of periostal callus formation were noticed on conventional radiographies. The rifampin treatment had prematurely been stopped by the patient due to considerable gastrointestinal sideeffects and weight loss.

At follow-up three and five months after nail exchange, there were no clinical signs of infection and the inflammatory parameters (WBC and CRP) were at normal levels. Conventional radiographies showed a rupture of the cranial cerclage wire, with secondary 
displacement of the greater trochanter (Figure 1D). Consolidation of the fracture however progressed well, so that full weight bearing was allowed at the follow-up consultation three months after nail exchange. The systemic antibiotic treatment with levofloxacin was maintained for a total of six months postoperatively. After one year, follow-up radiographs showed implant failure with a broken distal locking screw and non-union of the intertrochanteric fracture zone. Later on, the patient complained of increasing hip pain. Conventional radiographies and a computed tomography revealed progressive osteoarthritis of the hip, osteonecrosis of the femoral head in the main weight-bearing area, and a non-union of the trochanteric region (Figure 2A). Thus, total hip arthroplasty (THA) became necessary. Preoperative aspiration of the hip revealed no bacterial growth. The cephalomedullary nail and the cerclage wires were removed and THA performed through a transfemoral approach (Figure 2B) [5]. Only the usual perioperative antibiotic prophylaxis with cefuroxime was administered. Intraoperative tissue sampling was performed for microbiological workup and histology. No microbial growth was detected. The postoperative recovery was uneventful. At the last follow-up examination, two years after THA, the patient was pain free with an insufficiency of the hip abductors and a non-union of the greater trochanter. However no recurrence of infection was seen (Figure 2C \& 2D). Informed consent for publication of anonymised health-related data was obtained from the patient.
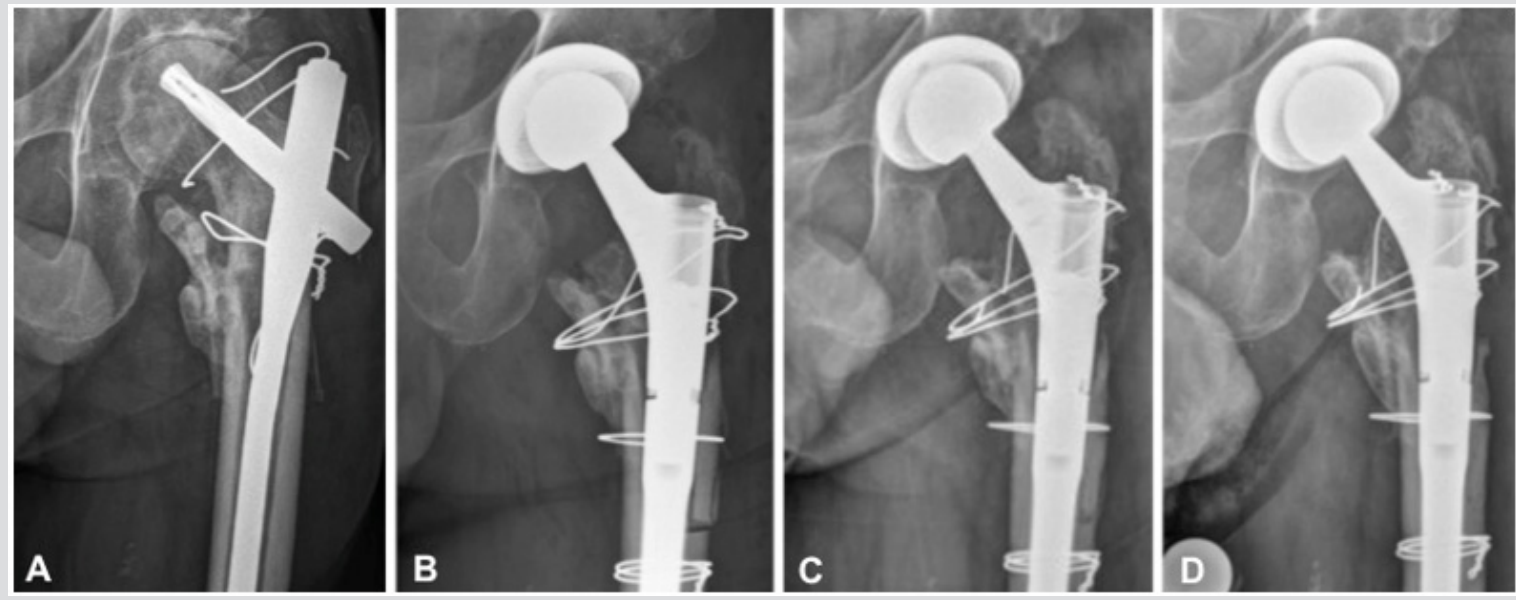

Figure 2: Picture A shows the development of osteoarthritis and an osteonecrosis of the femoral head during follow-up. Due to increasing left sided hip pain, total hip arthroplasty (THA) was performed, through a transfemoral approach and with a modular stem (Figure B). Follow-up radiographies showed a delayed consolidation of the greater trochanter (Figure C). At the last follow-up examination, 2 years after THA, the patient was pain free, but an insufficiency of the hip adductors and a nonunion of the greater trochanter persisted (Figure D). There were however no signs of recurrence of infection.

\section{Discussion}

We present a case of an implant-related infection with Bacillus spp after internal fixation of a closed trochanteric fracture with a cephalomedullary nail. To the best of our knowledge, this is only the second case reported in the literature showing a bacillus infection after surgical treatment of a closed fracture [6]. Infections with low virulence microorganisms, such as Bacillus spp, may be difficult to identify and may also be misinterpreted as contaminants [1,2]. Therefore, it is crucial to have an adequate and well-established diagnostic approach if implant-related infection is to be suspected. The procedure was not optimal in this case, and this hampered proper diagnosis and treatment. The current recommendations are reviewed, focusing on elementary steps in diagnostics that would permit to perform better. Bacillus spp are Gram positive, primarily aerobic, rod-shaped and spore-forming bacteria, which are widely spread in the environment and mostly found in the soil [7]. The spores are formed within the mother cell compartment, and they are extremely resistant to most environmental stress factors [8]. They are released when the mother cell lyses [8]. Spore resistance and dormancy is due to particular structure and components, such as an exosporium, an interspace and a coat layer, which are not found in growing cells [8-11]. Most Bacillus spp produce necrotizing toxins (cereolysin) and mimic the effect of staphylococcal and clostridial toxins [12]. Therefore, they can induce food poisoning, but are identified more frequently in osteomyelitis, bacteremia, pneumonia, endocarditis and meningitis, especially in immunocompromised patients [12]. Osteomyelitis caused by Bacillus spp is known to occur after open fractures, as this microorganism is ubiquitously present in the soil $[7,12,13]$. Most Bacillus spp however are of low virulence and are mostly discarded as non-pathogenic contaminants in clinical samples [2]. In presence of an implant, a detailed analysis of results is necessary before such conclusions can properly be drawn. An exception is B. anthracis, which might cause necrotizing and fulminant infections, particularly when airborne. In our case, both samples taken at the third revision showed growth of Bacillus spp. This was misinterpreted as contamination by the laboratory, considering only each sample individually. The patient was immunocompromised to a certain degree due to chronic 
alcohol abuse, which represents a known risk factor [12]. Even if the clinical signs were inconspicuous, postoperative wound oozing after implant-surgery should already alert the treating physician [2]. To ensure a better interpretation of the microbiological findings, proper sampling is crucial. Four to five tissue biopsies should be sampled in immediate proximity to the implant for microbiological analysis [14-16].

Tissue biopsies provide a higher sensitivity and specificity than swab cultures, particularly for detecting microorganisms of low virulence $[16,17]$. Prolonged incubation on various media under aerobic and anaerobic conditions is crucial to identify fastidious microorganisms, which might be present in implantrelated infections $[14,18,19]$. Whenever possible, antimicrobial therapy should be withheld for at least 2 weeks before sampling $[2,20,21]$. In our case, empirical antibiotic treatment interfered with microbiological workup and should not have been started preoperatively before sampling. Identification of phenotypically identical microorganisms in two or more samples is sufficient to diagnose infection $[1,14,21]$. Histology is very useful for a better differentiation between contamination and infection [22,23]. Granulocyte quantification thresholds have been defined for both periprosthetic joint infections and fracture-related infection, but technical specificities have to be considered for appropriate counts $[22,23]$. As surgery induces acute granulocytic inflammation, sampling has to be performed at the first revision, to avoid false positive results.

A fracture-related infection of a non-consolidated fracture corresponds to an osteomyelitis Cierny-Mader stage IV [4], where fracture stabilization is part of the management to treat the infection. As the infection already had to be considered chronified when the diagnosis was made, removal of the implant was indicated [2] However, Bacillus spp are microorganisms with a low virulence, allowing a one-stage exchange [2,21]. If a fracture already shows enough signs of consolidation, providing sufficient stability, simple implant removal alone may be considered. Suppressive therapy of the infection with hardware retention until fracture healing occurs represents another alternative approach [24]. Local application of vancomycin-loaded calcium sulphate is an adjunct that allows delivery of high and prolonged antibiotic concentrations at the site of infection, at concentrations even high enough to be active against staphylococcal biofilm [3]. Bacillus spp commonly produce beta-lactamases, but usually remain susceptible to a systemic antibiotic therapy with penicillin combined with a beta-lactamase inhibitor [12].

Fluorochinolones, aminoglycosides, clindamycin or vancomycin are alternative systemic antibiotic treatment options [12]. In our case we started postoperatively an intravenous antibiotic treatment with an aminopenicillin combined with a beta-lactamase inhibitor (amoxicillin/clavulanate), followed by an oral treatment with a fluorochinolone (levofloxacin). As sporulating bacterias are difficult to treat, an attempt was made to optimize the efficacy of the antibiotic treatment by adding rifampin, because of a particularly high sensitivity of the concerned bacteria antibiotic drug, expressed as a very low MIC. The addition of rifampin is otherwise only necessary to treat implant-related infections with Staphylococcus spp [21]. The antibiotic treatment was maintained for six months total in our case, to reduce the risk of late relapse of spore-forming bacteria, but no sufficient data is available in the literature regarding optimal duration of antibiotic treatment for such cases.

\section{Author Contributions}

S.E. und P.W. conceived the original idea, did the literature search and wrote the manuscript. S.M. and C.M. provided critical feedback and contributed to the discussion. C.M. performed the proofreading. All authors contributed to the final manuscript.

\section{Acknowledgment}

This work was supported by the Division of Orthopaedics and Traumatology of the Cantonal Hospital Winterthur, Winterthur, Switzerland.

\section{Conflict of Interest}

All authors declare that there are no conflicts of interest to report.

\section{Disclosure of funding}

No funding for this work was received from the National Institutes of Health (NIH), Wellcome Trust and the Howard Hughes Medical Institute (HHMI).

\section{References}

1. Atkins BL, Athanasou N, Deeks JJ, Crook DW, Simpson H, et al. (1998) Prospective evaluation of criteria for microbiological diagnosis of prosthetic-joint infection at revision arthroplasty. The OSIRIS Collaborative Study Group. J Clin Microbiol 36(10): 2932-2939.

2. Zimmerli W, Trampuz A, Ochsner PE (2004) Prosthetic-joint infections. N Engl J Med 351(16): 1645-54.

3. Wahl P, Guidi M, Benninger E, Ronn K, Gautier E, et al. (2017) The levels of vancomycin in the blood and the wound after the local treatment of bone and soft-tissue infection with antibiotic-loaded calcium sulphate as carrier material. Bone Joint J 99-B(11): 1537-1544.

4. Cierny G, Mader JT, Penninck JJ (2003) A clinical staging system for adult osteomyelitis. Clin Orthop Relat Res 414: 7-24.

5. Wagner M, Wagner H (1999) Der transfemorale Zugang zur Revision von Hüftendoprothesen. Operative Orthopädie und Traumatologie 11(4): 278-295.

6. Gallo PH, Melton Kreft R, Nistico L, Sotereanos NG, Sewecke JJ, et al. (2011) Demonstration of Bacillus cereus in orthopaedic-implant-related infection with use of a multi-primer polymerase chain reaction-mass spectrometric assay: report of two cases. J Bone Joint Surg Am 93(15): e85.

7. Dubouix A, Bonnet E, Alvarez M, Bensafi H, Archambaud M, et al. (2005) Bacillus cereus infections in Traumatology-Orthopaedics Department: retrospective investigation and improvement of healthcare practices. J Infect 50(1): 22-30.

8. Setlow P (2014) Germination of spores of Bacillus species: what we know and do not know. J Bacteriol. 196(7): 1297-1305. 
9. McKenney PT, Eichenberger P (2012) Dynamics of spore coat morphogenesis in Bacillus subtilis. Mol Microbiol.83(2): 245-260.

10. Henriques AO, Moran CP (2007) Structure, assembly, and function of the spore surface layers. Annu Rev Microbiol 61: 555-588.

11. Setlow P (2006) Spores of Bacillus subtilis: their resistance to and killing by radiation, heat and chemicals. J Appl Microbiol 101(3): 514-525.

12. Drobniewski FA (1993) Bacillus cereus and related species. Clin Microbiol Rev 6(4): 324-338.

13. Nguyen L, Minville V, Bensafi H, Laffosse JM, Chassery C, et al. (2007) [Open leg fracture with Bacillus cereus infection]. Ann Fr Anesth Reanim 26(9): 780-783.

14. Bemer P, Leger J, Tande D, Plouzeau C, Valentin AS, et al. (2016) How Many Samples and How Many Culture Media To Diagnose a Prosthetic Joint Infection: a Clinical and Microbiological Prospective Multicenter Study. J Clin Microbiol 54(2): 385-391.

15. Metsemakers WJ, Morgenstern M, McNally MA, Moriarty TF, McFadyen I, et al. (2018) Fracture-related infection: A consensus on definition from an international expert group. Injury 49(3): 505-510.

16. Font Vizcarra L, Garcia S, Martinez Pastor JC, Sierra JM, Soriano A, et al. (2010) Blood culture flasks for culturing synovial fluid in prosthetic joint infections. Clin Orthop Relat Res 468(8): 2238-2243.

17. Aggarwal VK, Higuera C, Deirmengian G, Parvizi J, Austin MS, et al. (2013) Swab cultures are not as effective as tissue cultures for diagnosis of periprosthetic joint infection. Clin Orthop Relat Res 471(10): 31963203.

ISSN: 2574-1241

DOI: 10.26717/BJSTR.2019.19.003274

Sebastian Ebert. Biomed J Sci \& Tech Res

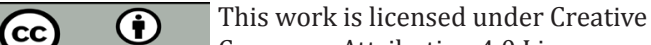

Submission Link: https://biomedres.us/submit-manuscript.php
18. Schwotzer N, Wahl P, Fracheboud D, Gautier E, Chuard C, et al. (2014) Optimal culture incubation time in orthopedic device-associated infections: a retrospective analysis of prolonged 14-day incubation. J Clin Microbiol 52(1): 61-66.

19. Schafer P, Fink B, Sandow D, Margull A, Berger I, et al. (2008) Prolonged bacterial culture to identify late periprosthetic joint infection: a promising strategy. Clin Infect Dis 47(11): 1403-1409.

20. Trampuz A, Piper KE, Jacobson MJ, Hanssen AD, Unni KK, et al. (2007) Sonication of removed hip and knee prostheses for diagnosis of infection. N Engl J Med 357(7): 654-663.

21. Osmon DR, Berbari EF, Berendt AR, Lew D, Zimmerli W, et al. (2013) Diagnosis and management of prosthetic joint infection: clinical practice guidelines by the Infectious Diseases Society of America. Clin Infect Dis 56(1): e1-e25

22. Morgenstern M, Athanasou NA, Ferguson JY, Metsemakers WJ, Atkins BL, et al. (2018) The value of quantitative histology in the diagnosis of fracture-related infection. Bone Joint J 100-B(7): 966-972.

23. Morawietz L, Tiddens O, Mueller M, Tohtz S, Gansukh T, et al. (2009) Twenty-three neutrophil granulocytes in 10 high-power fields is the best histopathological threshold to differentiate between aseptic and septic endoprosthesis loosening. Histopathology 54(7): 847-853.

24. Feihl S, Renz N, Schutz M, Muller M (2017) Antibiotic strategies in trauma surgery: Treatment of implant-associated infections. Unfallchirurg 120(6): 486-493.

$\begin{array}{ll}\text { BIOMEDICAL } & \text { Assets of Publishing with us } \\ \text { RESEARCHES } & \text { - Global archiving of articles } \\ & \text { - Immediate, unrestricted online access } \\ & \text { - }\end{array}$

